\title{
Diffuse Alveolar Hemorrhage (DAH) Associated with Severe Acute Respiratory Syndrome Coronavirus 2 (SARS-CoV-2): A Case Report
}

Haytham Wali ( $\sim$ haytham.a.wali@gmail.com )

Columbus Regional Healthcare System: Piedmont Healthcare Inc https://orcid.org/0000-0001-62793809

Deanne Tabb

Columbus Regional Healthcare System: Piedmont Healthcare Inc

Saeed A. Baloch

Columbus Regional Healthcare System: Piedmont Healthcare Inc

\section{Research Article}

Keywords: Diffuse alveolar hemorrhage, ANCA-associated vasculitis, glomerulonephritis, coronavirus disease-19

Posted Date: July 26th, 2021

DOI: https://doi.org/10.21203/rs.3.rs-555436/v1

License: (c) (i) This work is licensed under a Creative Commons Attribution 4.0 International License. Read Full License 


\section{Abstract}

Diffuse alveolar hemorrhage (DAH) is a rare syndrome resulting from the accumulation of intra-alveolar red blood cells (RBCs) originating most often from the alveolar capillaries and, less frequently, from precapillary arterioles or postcapillary venules. The causes of DAH can be divided into infectious and noninfectious. Severe acute respiratory syndrome coronavirus 2 (SARS-CoV-2) is a novel coronavirus that has not been previously identified in humans, and it is responsible for the coronavirus disease-19 (COVID19) infection. The long-term complications of COVID-19 are not entirely known and are still being investigated. Here, we present a case of DAH that is believed to be a consequence of COVID-19 infection.

\section{Introduction}

Diffuse alveolar hemorrhage (DAH), commonly recognized by the signs of anemia, hemoptysis, diffuse radiographic pulmonary infiltrates, and hypoxemic respiratory failure, is a rare syndrome resulting from the accumulation of intra-alveolar red blood cells (RBCs) originating most often from the alveolar capillaries and, less frequently, from precapillary arterioles or postcapillary venules [1-3]. The prognosis of DAH is poor, with hospital mortality ranging from $20 \%$ to over $50 \%$ [4]. The causes of DAH can be divided into infectious and noninfectious. Pulmonary infections include those caused by viruses, bacteria, fungi, and parasites [3]. In immunocompromised patients, the common infectious diseases that may cause DAH can consist of cytomegalovirus (CMV), adenovirus, invasive aspergillosis, Mycoplasma, Legionella, and Strongyloidiasis. However, in immunocompetent patients, the infections that may cause DAH can include influenza A (H1N1), dengue, leptospirosis, and malaria [5-14].

Severe acute respiratory syndrome coronavirus 2 (SARS-CoV-2) is a novel coronavirus that has not been previously identified in humans and is responsible for the coronavirus disease-19 (COVID-19) infection. The first case was identified in Wuhan, China, in December 2019, and it has spread to many countries around the world and was declared a global pandemic on March 11, 2020, by the World Health Organization (WHO). However, to our knowledge, the association between DAH and COVID-19 infection has not been reported. Here, we present a case of DAH that is believed to be a consequence of COVID-19 infection.

\section{Case Report}

This report describes a 26-year-old Caucasian female with no known past medical history who presented to the Piedmont Columbus Regional Midtown emergency department with a chief complaint of worsening shortness of breath and dyspnea on exertion. The patient was found to be positive for COVID19 around 25 days before this admission. The patient reported that she had isolated herself for ten days during that time and that her symptoms had improved. However, one week before the current admission, she started experiencing worsening shortness of breath and cough with hemoptysis. These symptoms prompted her visit to an emergency department, where she was subsequently diagnosed with atypical pneumonia and discharged on doxycycline [100 mg orally every 12 hours for five days]. 
The patient continued to worsen and was subsequently found to have generalized malaise, severe fatigue, non-productive cough, myalgias, fever, decreased appetite, and hemoptysis in the current admission. The patient had no family history of bleeding disorders. Her vital signs on admission were the following: temperature, $100.8^{\circ} \mathrm{F}\left(38.2^{\circ} \mathrm{C}\right)$; blood pressure, $128 / 72 \mathrm{mmHg}$; heart rate, 122 beats per minute; respiratory rate, 35 breaths per minute; and oxygen $\left(\mathrm{O}_{2}\right)$ saturation, $90 \%$ on room air, for which she required a $100 \%$ nonrebreather for oxygen support. An arterial blood gas (ABG) panel was performed and revealed the following: $\mathrm{pH}$ of 7.43 ; pCO2 of $32.9 \mathrm{mmHg}$; $\mathrm{pO} 2$ of $73.6 \mathrm{mmHg} ; \mathrm{HCO} 3$ of $21.9 \mathrm{mmol} / \mathrm{L}$. The patient had an elevated white blood cell (WBC) count of 11,200 cells/mcL [normal range, 4000-10500 cells $/ \mathrm{mcL}$ ], severe anemia with hemoglobin of $6.9 \mathrm{~g} / \mathrm{dL}$ [normal range, $12.0-16 \mathrm{~g} / \mathrm{dL}$ ], hematocrit of $20.6 \%$ [normal range, $36.0-48.0 \%$ ], and a platelet count of $43210^{3} / \mathrm{mcL}$ [normal range, $130-400$ $10^{3} / \mathrm{mcL}$ ]. The repeat COVID-19 test during this admission using the Xpert® Xpress SARS-CoV-2/Flu/RSV test (Cepheid ${ }^{\circledR}$, Sunnyvale, California, United States) and the SARS-COV-2 Immunoglobulin G (IgG) antibody were positive, which indicated that she was in the late stage of the infection.

The chest computed tomography (CT) scan was negative for pulmonary embolism (PE). However, it revealed extensive bilateral ground-glass opacities with confluent densities suggestive of multifocal pneumonia. Consequently, pulmonology and infectious diseases were consulted for evaluation and management. The patient was started on community-acquired pneumonia (CAP) treatment with ceftriaxone [ $1 \mathrm{~g}$ intravenously (IV) every 24 hours for five days] and azithromycin [500 mg IV every 24 hours for three days]. Additionally, there was strong suspicion for diffuse alveolar hemorrhage (DAH), most likely due to a COVID-19-associated vasculitis process, for which methylprednisolone [250 mg IV four times per day] was started.

A series of serologic tests were ordered to investigate the cause of DAH. The patient was found to be positive for perinuclear anti-neutrophil cytoplasmic antibodies (P-ANCA) [1:40 Titer; normal range, < 1:20 Titer]. She was also found to be negative for antinuclear antibody (ANA), anti-double-stranded deoxyribonucleic acid (anti-dsDNA), and cyclic citrullinated peptide (CCP) antibodies. Additionally, her total complement (CH50) was $49 \mathrm{U} / \mathrm{mL}$ [normal range, 31-60 U/mL], her $\mathrm{C} 3$ complement was $141 \mathrm{mg} / \mathrm{dL}$ [normal range, $90-180 \mathrm{mg} / \mathrm{dL}$ ], and her C4 complement was $16 \mathrm{mg} / \mathrm{dL}$ [normal range, $10-40 \mathrm{mg} / \mathrm{dL}$ ]. Based on these serologic findings, the patient was thought to have microscopic polyangiitis.

At the time of discharge, the patient had an oxygen saturation of $96 \%$ on room air and a respiratory rate of 18 breaths per minute with no evidence of ongoing hypoxia. The patient was prescribed a tapering course of prednisone [ $40 \mathrm{mg}$ orally twice daily for five days, then $30 \mathrm{mg}$ orally twice daily for five days, then $20 \mathrm{mg}$ orally twice daily for five days, then $10 \mathrm{mg}$ orally twice daily for five days, then $10 \mathrm{mg}$ orally once daily for five days] and advised to follow up with both pulmonology and her primary care physician.

Three days after discharge, the anti-glomerular basement membrane (anti-GBM) antibodies were found to be positive [4.5 Al; normal range, $<1.0 \mathrm{Al}$. In addition, the urinalysis (UA) obtained during admission confirmed the presence of blood and protein. The patient was informed about the positive results and advised to schedule an appointment with a nephrologist for further assessment. 
A renal biopsy was obtained during her visit with the nephrologist. The renal parenchyma available for light microscopic examination was represented by approximately $90 \%$ cortex and $10 \%$ medulla. Fortyeight glomeruli were present, none of which were globally sclerotic. Two glomeruli showed segmental rupture of the capillary loops associated with fibrinoid necrosis of the glomerular tuft and cellular crescent formation. Twenty-six additional glomeruli showed areas of segmental glomerulosclerosis, eleven of which were associated with fibro-cellular crescents and fifteen with fibrous crescents. The twenty uninvolved glomeruli showed no mesangial or endocapillary hypercellularity. The findings of the light microscopy can be seen in Fig. 1a and Fig. 1b.

The renal parenchyma available for immunofluorescence studies was entirely represented by cortex and contained nine glomeruli, none of which were globally sclerotic. The sections were stained for lgG, IgM, IgA, C3, complement 1q (C1q), albumin, fibrinogen, kappa, and lambda light chains. In addition, there was focal intense fibrinogen staining within the glomerular tuft. Additionally, there was mild mesangial finely granular staining for IgA (trace), IgG (2+), kappa (2+), and lambda (1+). All other stains were negative within the glomeruli. The findings of the immunofluorescence studies can be seen in Fig. 2a and Fig. $2 b$.

Based on the serology results mentioned above and the renal biopsy findings, the patient was determined to be having ANCA-associated vasculitis with glomerulonephritis and was started on methylprednisolone [500 mg IV daily for three days], followed by rituximab [375 mg/ $\mathrm{m}^{2} \mathrm{IV}$ once weekly for four weeks].

\section{Discussion}

A variety of diseases and conditions can be associated with the development of diffuse alveolar hemorrhage. One of the histopathologic patterns of DAH is pulmonary, or alveolar, capillaritis, which is characterized by the neutrophilic infiltration of the alveolar septa or the lung interstitium $[4,15]$. Although bronchoscopy with bronchoalveolar lavage (BAL) is indicated in patients suspected of having DAH to confirm the diagnosis [16], this procedure was not performed in our case due to the risk of aerosolization and spread of COVID-19 [17].

Anti-neutrophil cytoplasmic antibody (ANCA)-associated vasculitis (AAV) is a group of autoimmune diseases including granulomatosis with polyangiitis (GPA) (formerly Wegener's granulomatosis), eosinophilic granulomatosis with polyangiitis (formerly Churg-Strauss syndrome), and microscopic polyangiitis (MPA). These diseases are characterized by inflammatory cell infiltration causing necrosis of small blood vessels [18-20]. Our patient was thought to have diffuse alveolar hemorrhage caused by ANCA-associated vasculitis, but with the specific disease not wholly identified.

A positive ANCA test can indicate if DAH is due to GPA or microscopic polyangiitis. Cytoplasmic ANCA (CANCA), as determined by anti-proteinase 3 antibodies (anti-PR3), is most consistent with GPA. At the same time, perinuclear ANCA (P-ANCA) with positive anti-myeloperoxidase (anti-MPO) favors the diagnosis of microscopic polyangiitis or eosinophilic granulomatosis with polyangiitis syndrome [21]. Anti-GBM antibodies are more specific to an anti-GBM disease or Goodpasture's syndrome, an 
autoimmune disorder characterized by autoantibodies directed against the glomerular basement membrane. However, 60 to $80 \%$ of the patients with anti-GBM disease have clinical manifestations of pulmonary and renal disease [22]. In addition, patients with acute glomerulonephritis with or without pulmonary hemorrhage also may have GPA or MPA. Thus, the serum should be tested for ANCA as well as anti-GBM antibodies. Anti-GBM disease and systemic vasculitis not only have similar clinical manifestations, but between $10 \%$ and $50 \%$ of patients with the anti-GBM disease also test positively for ANCA (usually anti-MPO-ANCA) at the time of diagnosis and may have signs of a systemic vasculitis or a marked systemic inflammatory response. Based on our patient's serologic findings throughout her admission, she was diagnosed initially with microscopic polyangiitis. However, the discovery of the positive anti-GBM antibodies raised the suspicion for possible kidney involvement, which was subsequently confirmed by renal biopsy, even though the patient did not have any signs of kidney injury during her admission.

COVID-19 infection is classified into three stages: 1) early infection; 2) pulmonary involvement with or without hypoxia; 3 ) and the severe systemic hyper inflammation stage. The late stage of COVID-19 is characterized by the elevation of systemic inflammation markers [23]. It has been reported that inflammatory cytokines and biomarkers such as Interleukins (e.g., IL-2, IL-6, and IL-7), granulocyte colonystimulating factor, macrophage inflammatory protein 1-a, tumor necrosis factor (TNF)-a, C-reactive protein (CRP), ferritin, and D-dimer are significantly elevated during this stage [24]. In addition, some of these inflammatory markers, including IL-6, IL-8, and TNF-a, are elevated as well with ANCA-associated vasculitis [21]. Since our patient was in the late stage of her COVID-19 infection, which was supported by the time since the onset of her COVID-19 symptoms, her positive SARS-COV-2 IgG antibodies, and her inflammatory markers, it was possible that the elevation of inflammatory markers at the time of admission could have contributed to the incidence of ANCA-associated vasculitis and, subsequently, the diffuse alveolar hemorrhage.

\section{Conclusion}

We presented a rare case of diffuse alveolar hemorrhage in a patient following infection with SARS-COV2. The underlying cause is thought to be ANCA-associated vasculitis with glomerulonephritis. The association between COVID-19 infection and DAH is not fully known. However, the inflammatory process of COVID-19 infection may have a role in vasculitis, leading to DAH. Nevertheless, this case cannot establish a causal relationship between the two conditions, but it describes a clinical observation that might need to be considered when evaluating COVID-19 patients.

\section{Declarations}

\section{Funding}

The authors received no funding for this publication. 


\section{Conflicts of Interest/Competing Interests}

All the authors report no potential conflicts of interest.

\section{Availability of Data and Material}

Not applicable.

\section{Code Availability}

Not applicable.

\section{Authors' Contributions}

The first draft of the manuscript was written by Haytham Wali, and all authors commented on previous versions of the manuscript. All authors read and approved the final manuscript.

\section{Ethics Approval}

The Columbus Regional Research Integrity Panel has confirmed that no ethical approval is required.

\section{Consent to Publish}

The patient has consented to the submission of the case report to the journal.

\section{References}

1. Leatherman JW, Davies SF, Hoidal JR. Alveolar hemorrhage syndromes: diffuse microvascular lung hemorrhage in immune and idiopathic disorders. Med (Baltim). 1984;63(6):343-61.

2. de Prost N, Parrot A, Cuquemelle E, et al. Diffuse alveolar hemorrhage in immunocompetent patients: Etiologies and prognosis revisited. Respir Med. 2012;106(7):1021-32.

3. von Ranke FM, Zanetti G, Hochhegger B, Marchiori E. Infectious diseases causing diffuse alveolar hemorrhage in immunocompetent patients: a state-of-the-art review. Lung. 2013;191(1):9-18.

4. Lara AR, Schwarz MI. Diffuse alveolar hemorrhage. Chest. 2010;137(5):1164-71.

5. Ciledag A, Karnak D, Kayacan O. A butterfly-shaped alveolar hemorrhage caused by cytomegalovirus. Southeast Asian J Trop Med Public Health. 2010;41(4):900-3.

6. Mayeur N, Srairi M, Tetu L, Guilbeau Frugier C, Fourcade O, Dahan M. Lethal hemorrhagic alveolitis after adenovirus pneumonia in a lung transplant recipient. Heart Lung. 2012;41(4):401-3.

7. Agustí C, Ramirez J, Picado C, et al. Diffuse alveolar hemorrhage in allogeneic bone marrow transplantation. A postmortem study. Am J Respir Crit Care Med. 1995;151(4):1006-10.

8. Kane JR, Shenep JL, Krance RA, Hurwitz CA. Diffuse alveolar hemorrhage associated with mycoplasma hominis respiratory tract infection in a bone marrow transplant recipient. Chest. 1994;105(6):1891-2. 
9. Sundar KM, Pearce MJ. Diffuse alveolar hemorrhage due to Legionella pneumonia. Sarcoidosis Vasc Diffuse Lung Dis. 2004;21(2):158-9.

10. Agarwal VK, Khurana HS, Le HX, Mathisen G, Kamangar N. 30-year-old HIV-positive female with diffuse alveolar hemorrhage. J Intensive Care Med. 2009;24(3):200-4.

11. Gilbert CR, Vipul K, Baram M. Novel H1N1 influenza A viral infection complicated by alveolar hemorrhage. Respir Care. 2010;55(5):623-5.

12. Marchiori E, Ferreira JLN, Bittencourt CN, et al. Pulmonary hemorrhage syndrome associated with dengue fever, high-resolution computed tomography findings: a case report. Orphanet J Rare Dis. 2009;4:8.

13. Luks AM, Lakshminarayanan S, Hirschmann JV. Leptospirosis presenting as diffuse alveolar hemorrhage. Chest. 2003;123(2):639-43.

14. Corne $P$, Landreau L, Moulaire V, Jonquet $O$. Intra-alveolar hemorrhage during Plasmodium falciparum malarial crisis. Presse Med. 2001;30(30):1499.

15. Park MS. Diffuse alveolar hemorrhage. Tuberc Respir Dis. 2013;74(4):151.

16. loachimescu OC, Stoller JK. Diffuse alveolar hemorrhage: diagnosing it and finding the cause. Cleve Clin J Med. 2008;75(4):258, 260, 264-265 passim.

17. Krall J, Ali M, Maslonka M, Pickens A, Bellinger C. Bronchoscopy in the covid-19 era. Clinical Pulmonary Medicine. 2020;27(6):198-202.

18. Lazarus B, John GT, O'Callaghan C, Ranganathan D. Recent advances in anti-neutrophil cytoplasmic antibody-associated vasculitis. Indian J Nephrol. 2016;26(2):86-96.

19. Yates M, Watts R. ANCA-associated vasculitis. Clin Med (Lond). 2017;17(1):60-4.

20. Hunter RW, Welsh N, Farrah TE, Gallacher PJ, Dhaun N. ANCA associated vasculitis. BMJ. 2020;369:m1070.

21. Kronbichler A, Lee KH, Denicolò S, et al. Immunopathogenesis of ANCA-associated vasculitis. Int J Mol Sci. 2020;21(19).

22. Greco A, Rizzo MI, De Virgilio A, et al. Goodpasture's syndrome: A clinical update. Autoimmun Rev. 2015;14(3):246-53.

23. Segelmark M, Hellmark T, Wieslander J. The prognostic significance in Goodpasture's disease of specificity, titre and affinity of anti-glomerular-basement-membrane antibodies. Nephron Clin Pract. 2003;94:c59.

24. Kalluri R, Meyers K, Mogyorosi A, et al. Goodpasture syndrome involving overlap with Wegener's granulomatosis and anti-glomerular basement membrane disease. J Am Soc Nephrol. 1997;8:1795.

25. Levy JB, Hammad T, Coulthart A, et al. Clinical features and outcome of patients with both ANCA and anti-GBM antibodies. Kidney Int. 2004;66:1535.

26. McAdoo SP, Tanna A, Hru' ${ }^{1}$ ková $Z$, et al. Patients double-seropositive for ANCA and anti-GBM antibodies have varied renal survival, frequency of relapse, and outcomes compared to singleseropositive patients. Kidney Int. 2017;92:693. 
27. Siddiqi HK, Mehra MR. COVID-19 illness in native and immunosuppressed states: A clinicaltherapeutic staging proposal. The Journal of Heart Lung Transplantation. 2020;39(5):405-7.

28. Wu C, Chen X, Cai Y, et al. Risk factors associated with acute respiratory distress syndrome and death in patients with coronavirus disease 2019 pneumonia in Wuhan, China. JAMA Intern Med. 2020;180(7):934-43.

\section{Figures}
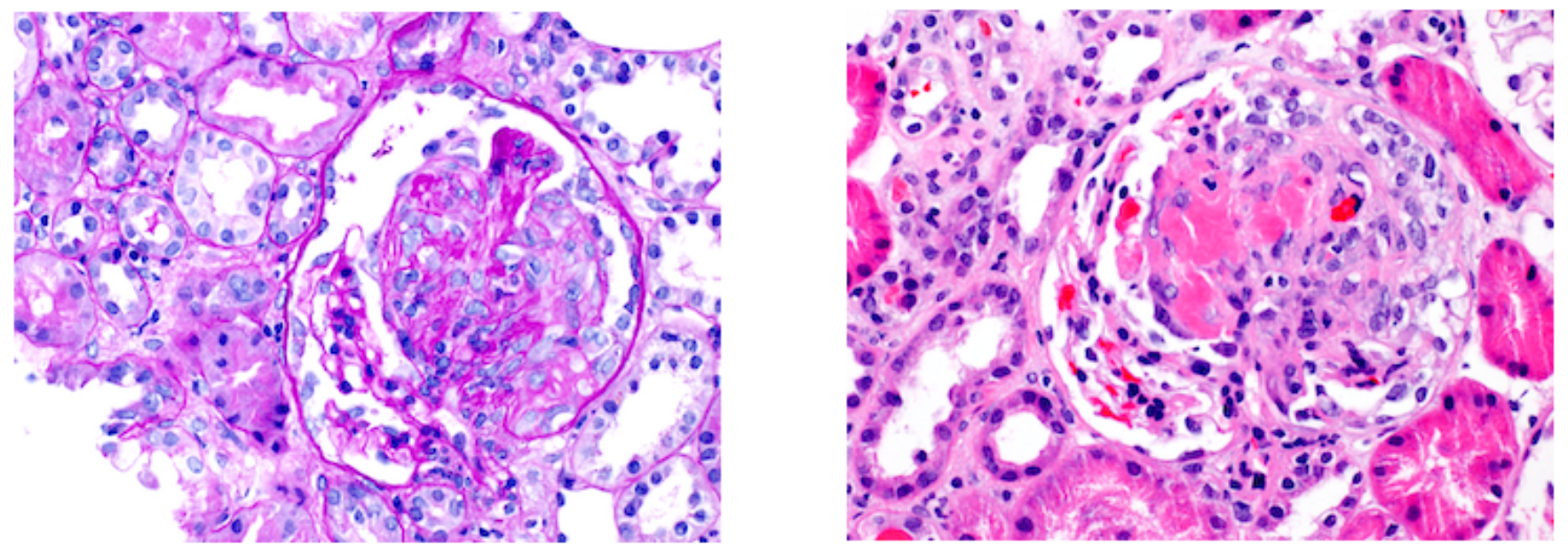

\section{Figure 1}

1a Light microscopy of the renal biopsy showing fibrocellular crescent. 1b Light microscopy of the renal biopsy showing fibrinoid necrosis. 

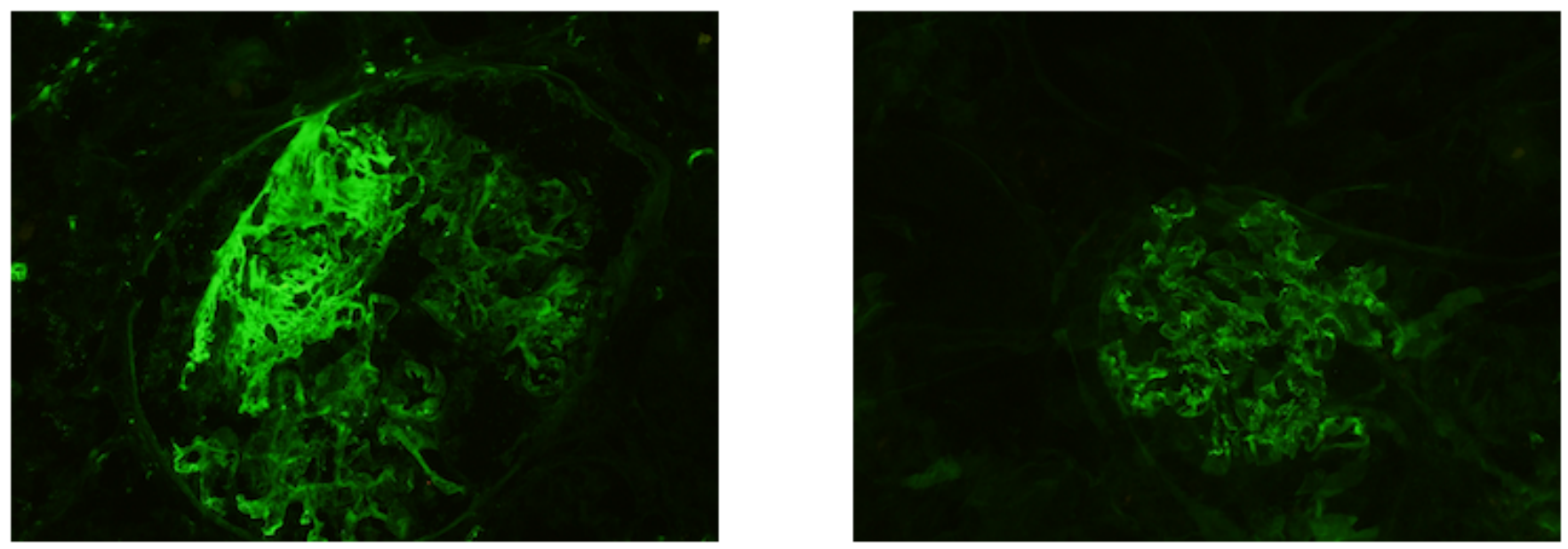

Figure 2

2a The immunofluorescence stain of the fibrinogen in the renal biopsy. $2 b$ The immunofluorescence stain of the immunoglobulin $\mathrm{G}(\mathrm{IgG})$ in the renal biopsy. 